\title{
Universiteit
}

Leiden

The Netherlands

\section{Identification of a radical intermediate in the enzymatic reduction of oxygen by small laccase}

Tepper, A.W.J.W.; Milikisyants, S.; Sottini, S.; Vijgenboom, E.; Groenen, E.J.J.; Canters, G.W.

\section{Citation}

Tepper, A. W. J. W., Milikisyants, S., Sottini, S., Vijgenboom, E., Groenen, E. J. J., \& Canters, G. W. (2009). Identification of a radical intermediate in the enzymatic reduction of oxygen by small laccase. Journal of The American Chemical Society, 131(33), 11680-11682. doi:10.1021/ja900751c

Version: Publisher's Version

License: $\quad$ Licensed under Article 25fa Copyright Act/Law (Amendment Taverne)

Downloaded from: https://hdl.handle.net/1887/3239369

Note: To cite this publication please use the final published version (if applicable). 


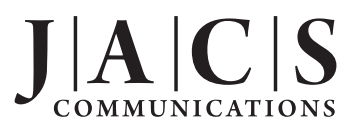

Published on Web 07/31/2009

\title{
Identification of a Radical Intermediate in the Enzymatic Reduction of Oxygen by a Small Laccase
}

\author{
Armand W.J.W. Tepper, ${ }^{\dagger}$ Sergey Milikisyants, ${ }^{\ddagger}$ Silvia Sottini, ${ }^{\ddagger}$ Erik Vijgenboom, ${ }^{\dagger}$ \\ Edgar J.J. Groenen, ${ }^{\ddagger}$ and Gerard W. Canters ${ }^{\ddagger, \dagger, *}$ \\ Leiden Institute of Chemistry and Leiden Institute of Physics, Leiden University, Leiden, The Netherlands
}

Received January 30, 2009; E-mail: canters@ chem.leidenuniv.nl

The recently described small laccase (SLAC) from Streptomyces coelicolor belongs to the family of multicopper oxidases (MCOs) and represents a new subclass of the laccases. ${ }^{1}$ The present work deals with a new transient biradical species that has been identified during the turnover of SLAC.

MCOs catalyze the oxidation of a variety of organic and inorganic substrates by molecular oxygen. Reducing equivalents are accepted one by one by a type- 1 (T1) Cu center and transferred to a trinuclear center (TNC), which consists of a type-2 (T2) $\mathrm{Cu}$ site and a binuclear type-3 (T3) $\mathrm{Cu}$ site (Figure 1a). Oxygen is converted into water at the TNC. Messerschmidt et al. ${ }^{2}$ provided the first detailed structural information on an MCO. They showed that ascorbate oxidase (AO) is composed of three cupredoxin domains, with domain 1 containing the T1 site and the TNC being located at the interface of domains 1 and 3. Domain 2 connects the other two domains and helps shape the access and exit channels to the TNC. It was later shown that this domain organization is the basic pattern along which the laccases are built, too. ${ }^{3}$

A large body of kinetic, spectroscopic, and structural work has been amassed over the past 40 years dealing with the mechanism of the oxygen-to-water conversion. Most of this work has focused on laccases. ${ }^{3,4}$ The first step in the reduction of oxygen by a fourelectron-reduced laccase consists of two-electron oxidation of the $\mathrm{T} 3$ site, with concomitant production of a peroxide intermediate, followed by a one-electron step whereby the oxygen-oxygen bond is broken and an oxygen radical is produced. ${ }^{5,6}$ Later, this threeelectron intermediate was found to correspond to the product of a four-electron oxygen reduction, the spectroscopic properties of which are compatible with a fully oxidized TNC in which the $\mathrm{Cu}^{2+}$ ions are exchange-coupled by hydroxy or water bridges. ${ }^{4,7}$

SLAC differs from the regular laccases in that domain 2 appears to be missing in the molecular architecture, which thus consists of only two cupredoxin domains. Because of this difference, we thought it of interest to investigate the enzyme mechanism of SLAC. Optical, electron paramagnetic resonance (EPR), and kinetic studies have shown that a transient species can be identified during enzymatic turnover of the enzyme as well as in single-turnover experiments of the wild-type (wt) and type-1-depleted (T1D) forms of the SLAC.

The optical absorption spectrum [see the Supporting Information (SI) for experimental details] of oxidized wt SLAC in the resting state (Figure 1b) exhibits the typical MCO features: the strong absorption at $590 \mathrm{~nm}\left(\varepsilon=4.4 \mathrm{mM}^{-1} \mathrm{~cm}^{-1}\right)$ and the shoulder extending into the near-IR are both associated with the T1 copper; the absorption at $330 \mathrm{~nm}\left(\varepsilon=3.2 \mathrm{mM}^{-1} \mathrm{~cm}^{-1}\right)$ is associated with the trinuclear cluster and has been ascribed to a charge-transfer transition from a bridging hydroxy group to the dinuclear $\mathrm{Cu}$ site

\footnotetext{
' Leiden Institute of Chemistry.

* Leiden Institute of Physics.
}

in the $\mathrm{T} 3$ center. ${ }^{4,7}$ As shown elsewhere, the EPR spectrum of the resting wt enzyme is dominated by the contribution from the T1 copper. $^{1}$

In a steady-state turnover experiment, $2 \mathrm{mM}$ ascorbate was added to a $65 \mu \mathrm{M}$ solution of fully oxidized SLAC in the presence of $260 \mu \mathrm{M} \mathrm{O}_{2}$. Enzymatic turnover resulted in the gradual decrease of the ascorbate absorption at $260 \mathrm{~nm}$ (not shown). In the optical spectrum, a band grew in at $420 \mathrm{~nm}$, reached a maximum in the steady state in $\sim 16 \mathrm{~s}$ (Figure 1c), and disappeared at the end of the reaction (when all of the oxygen had been consumed) along with the 330 and $590 \mathrm{~nm}$ bands (not shown). We note that during steady-state turnover, the latter bands, which are characteristic of the oxidized T3 and T1 sites, respectively, did not change in intensity, indicating that these sites remained oxidized. This means that oxidation of the ascorbate is the rate-determining step in the enzymatic reaction.

Next, in a single-turnover experiment in which $8 \mu \mathrm{M}$ ascorbatereduced SLAC was mixed in a stopped-flow apparatus with an excess of oxygen $(130 \mu \mathrm{M})$, the $420 \mathrm{~nm}$ band appeared again, next to the 330 and $590 \mathrm{~nm}$ bands (Figure 1d). The spectral changes occurred monoexponentially, all with the same rate constant of 23 $\pm 1 \mathrm{~s}^{-1}$. After reaching a maximum, the $420 \mathrm{~nm}$ band disappeared within $\sim 100 \mathrm{~s}$.

To further identify the source of the transient signal, we took recourse to inactivation of the $\mathrm{T} 1$ site. $^{3,4,7,8}$ This allowed us to investigate the possible connection of the $420 \mathrm{~nm}$ band with the TNC because it abolished the contributions of the T1 site to the optical and EPR spectra of the enzyme, making the TNC features stand out more clearly. Also, it may stabilize a possible threeelectron-reduced intermediate, allowing for its characterization. ${ }^{9}$

A variant of SLAC in which the $\mathrm{T} 1 \mathrm{Cu}$-coordinating Cys was replaced by Ser (C288S) was prepared. Spectroscopic and biochemical characterization (see Figure $1 \mathrm{~b}$ and the SI) showed that the T1 site was empty while the TNC was intact. The reaction kinetics of this variant was studied by optical as well as EPR spectroscopy. Mixing fully reduced T1D SLAC with excess $\mathrm{O}_{2}$ at $\mathrm{pH} 6.8$ again resulted in the appearance of the optical transition at $330 \mathrm{~nm}$ and the optical absorption band of the transient at $\sim 420$ $\mathrm{nm}$, both with a monoexponential rate constant of $23 \pm 1 \mathrm{~s}^{-1}$ (Figure 1e), identical to that observed in the single-turnover experiment with wt SLAC (see above). The $420 \mathrm{~nm}$ band slowly decayed biexponentially with rate constants of $6.2 \pm 0.6 \mathrm{~h}^{-1}$ and $0.9 \pm 0.2$ $\mathrm{h}^{-1}$ (Figure 1f). For the EPR experiment, a reduced T1D sample was mixed with excess $\mathrm{O}_{2}$, after which it was lowered into liquid $\mathrm{N}_{2}$ in less than $10 \mathrm{~s}$ and its X-band EPR spectrum was taken. The sample was then warmed to room temperature and, after a certain time interval, frozen and measured again. The EPR spectrum consisted of a $g=2$ signal (Figure 2a) and a signal in the $g=4$ region (half-field signal) (Figure $2 b$ ), both of which changed with time. It was found (see the SI) that the spectra in the $g=2$ region 

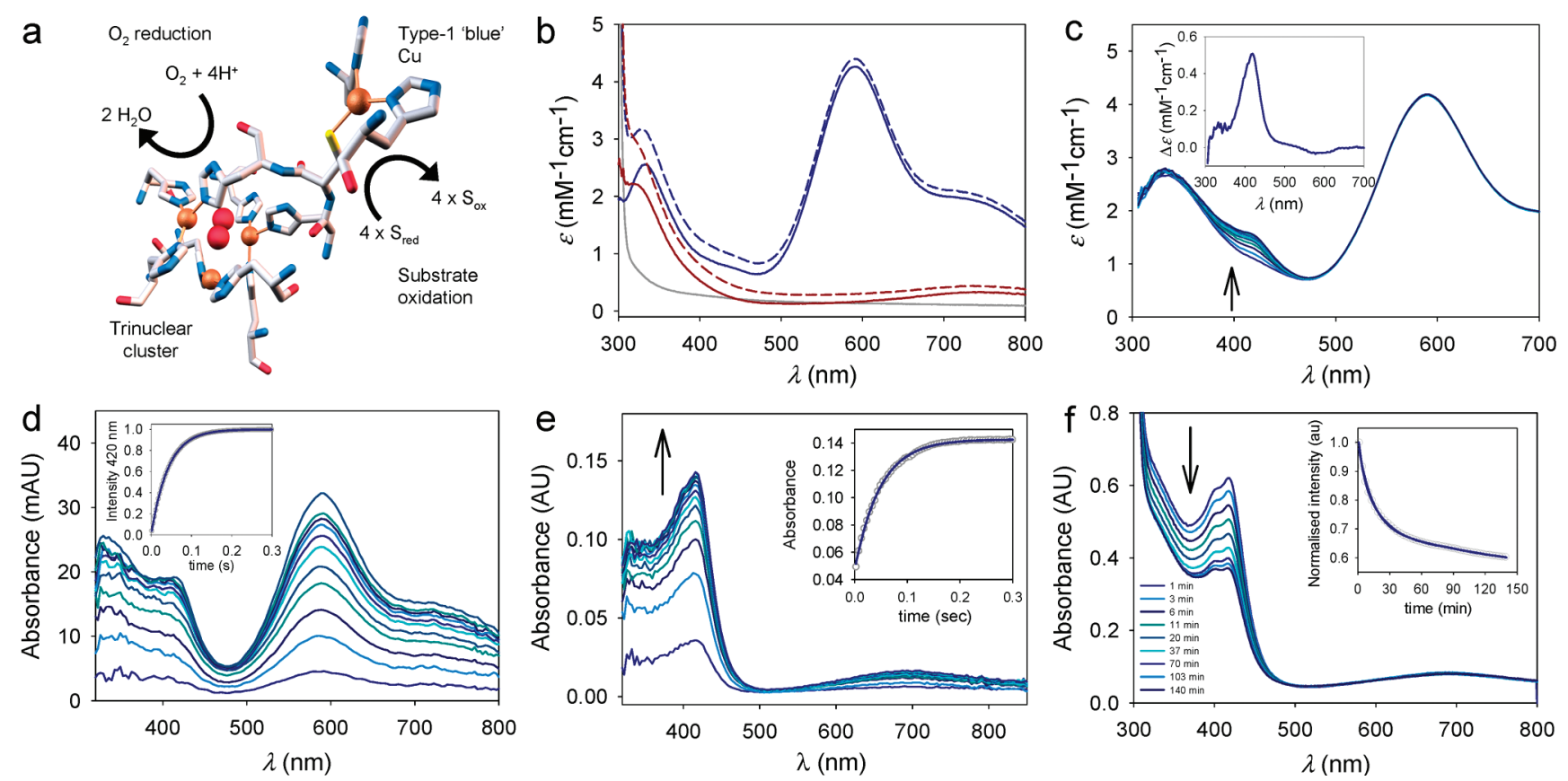

Figure 1. Optical spectroscopy of wt and T1D SLACs. (a) MCO active site from Melanocarpus albomyces laccase (PDB entry 1GW0). Color code: Cu, gold; $\mathrm{O}_{2}$, red; protein, stick representation with conventional color code. (b) Optical absorption spectra of oxidized wt (blue, dashed) and T1D (red, dashed) SLACs and reduced (gray) wt and T1D SLACs (coinciding). Solid lines are oxidized-minus-reduced difference spectra [100 mM $\mathrm{P}_{\mathrm{i}}(\mathrm{pH} 6.80)$ ), RT]. (c) Optical absorption spectra of wt SLAC during turnover of ascorbate. Spectra were recorded every second after mixing $65 \mu \mathrm{M}$ fully oxidized wt SLAC with $2 \mathrm{mM}$ ascorbate in the presence of $0.26 \mathrm{mM} \mathrm{O}_{2}\left[0.1 \mathrm{M} \mathrm{P}_{\mathrm{i}}(\mathrm{pH} 6.8), \mathrm{RT}\right]$ in a stopped-flow apparatus over a period of $16 \mathrm{~s}$. The spectra were corrected for the contribution of the reduced enzyme. Inset: difference between spectra recorded at $t=14 \mathrm{~s}$ and $t=2 \mathrm{~s}$. (d) Single turnover of wt SLAC. Spectra were recorded every $3.2 \mathrm{~ms}$ after mixing $8 \mu \mathrm{M}$ of ascorbate-reduced wt SLAC with excess $\mathrm{O}_{2}(0.13 \mathrm{mM})$ in a stopped-flow apparatus (not all of the spectra are shown). The line at the top is the spectrum observed at $t=1 \mathrm{~s}$. The spectra were corrected for the contribution of the reduced enzyme. Inset: absorbance at $420 \mathrm{~nm}$ vs time. At all wavelengths, the absorption increased monoexponentially (solid line) with a rate constant of $23 \pm 1 \mathrm{~s}^{-1}$. (e) Single turnover of T1D SLAC. Spectra were recorded every $20 \mathrm{~ms}$ after mixing $83 \mu \mathrm{M}$ of dithionite-reduced T1D SLAC with excess $\mathrm{O}_{2}(0.13 \mathrm{mM})$ in a stopped-flow apparatus (not all of the spectra are shown). The spectra were corrected for the contribution of the reduced enzyme. Inset: absorbance at $420 \mathrm{~nm}$ vs time. At all wavelengths, the absorption increased monoexponentially (solid line) with a rate constant of $23 \pm 1 \mathrm{~s}^{-1}$. (f) Same as (e) but on a longer time scale. Conditions: $0.26 \mathrm{mM}$ reduced T1D SLAC, saturation with $\mathrm{O}_{2}$ at $t=0$. The first spectrum was taken at $t=1 \mathrm{~min}$ (top). Inset: absorbance at $420 \mathrm{~nm}$ vs time. The solid line is a biexponential fit (rate constants: 6.2 and $0.9 \mathrm{~h}^{-1}$ )

correspond to two species, a transient one appearing within the dead time of the EPR experiment (i.e., seconds) (Figure 2c) and decaying with time while the other one, with the EPR features of a regular type- $2 \mathrm{Cu}$ site, grew in. After reaching a maximum, the latter stayed constant for the duration of the experiment $(4 \mathrm{~h})$. For the present study, we focused on the transient species.

The intensity of the transient species decayed biexponentially with time constants of $10 \pm 1 \mathrm{~h}^{-1}$ and $0.9 \pm 0.4 \mathrm{~h}^{-1}$ (Figure 2a, inset). The signal-to-noise ratio of the half-field signal for delays longer than 20 min was too low to enable a quantitative analysis over the same time window $(4 \mathrm{~h})$ as used for the $g=2$ signal. The initial decay of the $g=4$ signal, however, was the same as that of the $g=2$ signal (Figure 2a, inset), and both signals were assigned to the same species. Furthermore, the decay constants measured by EPR spectroscopy are similar to those measured for the 420 nm optical transition, ${ }^{10}$ supporting the conclusion that the EPR and optical signatures derive from the same species.

To what does the transient species correspond? The $g=4 \mathrm{EPR}$ signal is indicative of a biradical triplet state. Its splitting $(\sim 10$ $\mathrm{mT}$ ) into four equidistant lines apparently derives from the interaction with a $\mathrm{Cu}$ nucleus. Similar half-field signals have been observed for paramagnetically labeled coordination compounds of $\mathrm{Cu} .{ }^{11-13}$ One of the spins, therefore, resides on a $\mathrm{Cu}$ in the TNC. Its distance to the second spin can be inferred from the intensity of the half-field signal. ${ }^{11,14}$ In the present case, the ratio of the intensities of the $g=4$ and $g=2$ signals was $(1: 1000) \pm 10 \%$, pointing to a distance of $5 \pm 0.2 \AA$. $^{11,15}$
For the location of the second spin, various possibilities were considered. The spin might reside on an oxygen-derived radical, but incubation of reduced T1D SLAC with $95 \%$ enriched ${ }^{17} \mathrm{O}_{2}$ resulted in spectra that were indistinguishable from the spectra obtained by incubation with air (not shown). The spin might reside on another $\mathrm{Cu}$ atom in the TNC, but the half-field signal in that case would exhibit a fine structure consisting of many more than the four hyperfine lines that we actually observed (Figure $2 b) .{ }^{15-17}$ It is more likely, therefore, that the second unpaired spin originates from a protein-derived radical. The optical fingerprint (Figure 1f) would be compatible with, for instance, a tyrosine radical. ${ }^{18,19}$

$\mathrm{A} \mathrm{Cu}^{2+}$-tyrosyl radical pair with optical characteristics similar to those observed here has been found in the activated form of galactose oxidase and in model compounds. ${ }^{12,13,18,19}$ Although the spin-spin coupling in galactose oxidase is antiferromagnetic, leading to an EPR-silent $S=0$ state, some $\mathrm{Cu}^{2+}$-phenoxyl model compounds are reported to have ferromagnetic coupling and $S=1$ ground states. ${ }^{12,18}$ Moreover, Wieghardt and co-workers ${ }^{13}$ have demonstrated that the sign of the exchange coupling sensitively depends on the configuration of the $\mathrm{Cu}$-phenoxyl moiety and may change from antiferro- to ferromagnetic with a change in the orientation of the phenoxyl radical with respect to the $\mathrm{Cu}$ orbital that carries the unpaired spin. In regard to the assumption that the second spin is localized on a phenoxyl oxygen, the spin-spin distance calculated above $(5 \pm 0.2 \AA)$ seems too large to be compatible with a $\mathrm{Cu}$-oxygen bond; however, spin delocalization over the phenyl moiety would increase the effective spin-spin 

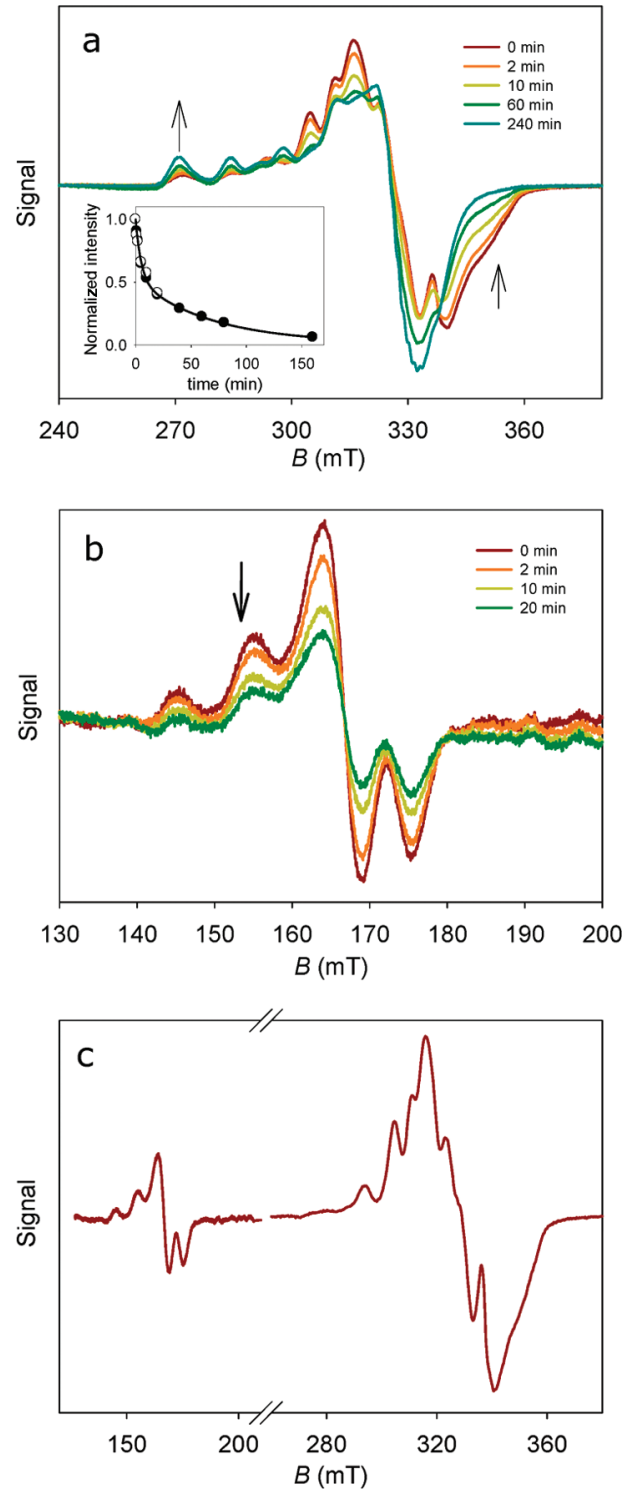

Figure 2. EPR spectroscopy. (a) X-band EPR spectra at $T=40 \mathrm{~K}$ in the $g=2$ region recorded at various time intervals after incubation of $0.7 \mathrm{mM}$ reduced T1D SLAC $\left[0.1 \mathrm{M} \mathrm{P}_{\mathrm{i}}\left(\mathrm{pH}\right.\right.$ 6.8)] with excess $\mathrm{O}_{2}$. Inset: normalized intensity of the intermediate as a function of time in the $g=2(\bullet)$ and $g$ $=4(\mathrm{O})$ regions. The solid line is a biexponential fit (rate constants: $10 \mathrm{~h}^{-1}$ and $0.9 \mathrm{~h}^{-1}$ ). (b) Spectra in the $g=4$ region. The spectrum recorded at $240 \mathrm{~min}$ was used as the reference to remove the background contribution to the signal. (c) EPR spectrum of the transient species as derived from (a) and (b). It should be noted that the vertical scales of the $g=2$ and $g=4$ signals are different.

distance. We must await structural data for the SLAC to see if this is a realistic possibility. ${ }^{20}$

We note that the EPR observations indicate that the electrons are exchange-coupled. If the electron spins were only weakly dipolar-coupled, one would expect an intense EPR line from the organic radical around the free-electron $g$ value in the W-band spectrum. However, no such signal was observed (Figure S3).

The coupling must surpass the $g$ anisotropy, which amounts to $\sim 3 \mathrm{GHz}$ or $0.1 \mathrm{~cm}^{-1}$. A small orbital overlap between the $\mathrm{T} 2 \mathrm{Cu}^{2+}$ and the radical would be sufficient to produce an exchange interaction of this magnitude.
It is noteworthy that the oxidation by molecular oxygen of reduced T1D SLAC, which contains only three reducing equivalents (one electron on each $\mathrm{Cu}$ in the TNC), apparently leads to a fourelectron-oxidized state. The fourth electron appears to be provided by the protein itself. The intermediate also occurs during turnover of the wt enzyme, showing that it may play a role in the catalytic cycle. It might prevent the occurrence of a reactive and deleterious three-electron-reduced oxygen species and may even play a role in the conversion of organic substrates that function as electron donors for the enzyme. It remains to be seen whether the present findings are characteristic of a whole class of MCOs, i.e., the small laccases, or merely represent a singularity in the general pattern found for the regular laccases.

Acknowledgment. We thank The Netherlands Organization for Scientific Research (NWO-CW) and its programme "From Molecule to Cell" as well as the Foundation for Fundamental Research on Matter (FOM) for support. We thank Dr. M. Machczynski for assistance with the preparation of the T1D mutant.

Supporting Information Available: Experimental procedures, SDS PAGE of wt SLAC and the T1D mutant, and EPR spectra of resting T1D SLAC (X-band) and the biradical intermediate (W-band). This material is available free of charge via the Internet at http://pubs.acs.org.

\section{References}

(1) Machczynski, M. C.; Vijgenboom, E.; Samyn, B.; Canters, G. W. Protein Sci. 2004, 13, 2388.

(2) Messerschmidt, A; Ladenstein, R. Huber, R; Bolognesi, M.; Avigliano, L.; Petruzzelli, R.; Rossi, A.; Finazziagro, A. J. Mol. Biol. 1992, 224, 179.

(3) Messerschmidt, A. Multi-Copper Oxidases; World Scientific Publishing: Singapore, 1997.

(4) Solomon, E. I.; Augustine, A. J.; Yoon, J. Dalton Trans. 2008, 3921

(5) Aasa, R.; Branden, R.; Deinum, J.; Malmstrom, B. G.; Reinhammar, B.; Vanngard, T. FEBS Lett. 1976, 61, 115.

(6) Andreasson, L. E.; Branden, R.; Reinhammar, B. Biochim. Biophys. Acta 1976, 438, 370 .

(7) Solomon, E. I.; Chen, P.; Metz, M.; Lee, S. K.; Palmer, A. E. Angew. Chem., Int. Ed. 2001, 40, 4570.

(8) Morie-Bebel, M. M.; McMillin, D. R.; Antholine, W. E. Biochem. J. 1986, $235,415$.

(9) Cole, J. L.; Ballou, D. P.; Solomon, E. I. J. Am. Chem. Soc. 1991, 113, 8544.

(10) The small difference is primarily due to the different circumstances under which the optical and EPR samples had to be prepared and studied. In particular, the time scale of the reaction in the EPR experiment is less well defined because of the freeze-thaw cycles involved.

(11) Eaton, S. S.; More, K. M.; Sawant, B. M.; Eaton, G. R. J. Am. Chem. Soc. 1983, 105, 6560 .

(12) Michel, F.; Thomas, F.; Hamman, S.; Philouze, C.; Saint-Aman, E.; Pierre, J. L. Eur. J. Inorg. Chem. 2006, 3684.

(13) Muller, J.; Weyhermuller, T.; Bill, E.; Hildebrandt, P.; Ould-Moussa, L.; Glaser, T.; Wieghardt, K. Angew. Chem, Int. Ed. 1998, 37, 616.

(14) Eaton et al. ${ }^{11}$ have shown that if the exchange interaction between the spins is isotropic, the integrated intensity of the half-field signal divided by that of the $g=2$ signal is given by $I_{\mathrm{g}=4} / I_{\mathrm{g}=2}=\left(A / r^{6}\right)(9.1 / v)$, where $v$ is the spectrometer frequency $(\mathrm{GHz}), r$ is the spin-spin distance $(\AA)$, and $A \approx$ 20 is a numerical constant.

(15) Smith, T. D.; Pilbrow, J. R. Coord. Chem. Rev. 1974, 13, 173.

(16) Boas, J. F.; Dunhill, R. H.; Pilbrow, J. R.; Srivasta, R. C.; Smith, T. D. J. Chem. Soc. A 1969, 94.

(17) Eaton, S. S.; Eaton, G. R.; Chang, C. K. J. Am. Chem. Soc. 1985, 107, 3177.

(18) Sokolowski, A.; Leutbecher, H.; Weyhermuller, T.; Schnepf, R.; Both, E.; Bill, E.; Hildebrandt, P.; Wieghardt, K. J. Biol. Inorg. Chem. 1997, 2, 444.

(19) Whittaker, M. M.; Whittaker, J. W. J. Biol. Chem. 1988, 263, 6074

(20) After completion of the manuscript, the recently published X-ray structure of SLAC came to our attention ( Skalova, T.; Dohnalek, J.; Ostergaard, L. H.; Ostergaard, P. R.; Kolenko, P.; Duskova, J.; Stepankova, A.; Hasek, J. J. Mol. Biol. 2009, 385, 1165. ). In this structure, the oxygen of Tyr 108 is located at a distance of $4.6 \AA$ from the T2 Cu at the end of a solventaccessible channel that leads to the TNC. This residue is an obvious candidate as the source of the radical. 